\title{
ANÁLISE DE QUESTÕES DE FÍSICA DO ENEM PELA TAXONOMIA DE BLOOM REVISADA
}

RESUMO: O Exame Nacional do Ensino Médio (Enem), instituído em 1998, ganhou notoriedade em 2005 como mecanismo de acesso ao Programa Universidade para Todos (ProUni) e consagrou-se a partir de 2009 como mecanismo de acesso ao Sistema de Seleção Unificado (Sisu), superando 7 (sete) milhões de inscritos em 2013. O presente trabalho analisa, na perspectiva da Taxonomia de Bloom Revisada (TBR), 105 questões de física do Enem aplicadas entre 2009 e 2013, almejando compreender as dimensões do conhecimento e do processo cognitivo da TBR privilegiadas no Exame. Entre as dimensões do conhecimento, destacam-se o conhecimento conceitual (56\%), seguido pelo conhecimento procedural (31\%), e, entre os processos cognitivos, destacam-se entender (49\%) e aplicar (23\%), indicando que o exame enfatiza domínios de complexidade intermediários nas dimensões analisadas.

Palavras-chave: Enem. Taxonomia de Bloom Revisada. Ensino de física.

\section{ANALYSIS OF QUESTIONS OF PHYSICS FOR THE NATIONAL STANDARD EXAM OF MIDDLE-HIGH SCHOOL (ENEM) ACCORDING TO REVISED BLOOM'S TAXONOMY}

ABSTRACT: The National Standard High School Exam (ENEM), established in 1998, gained prominence in 2005, when it became the mechanism to get into the high education program University to Everybody (PROUNI) and even more notorious in 2009 as an entrance exam to the United Access System (SISU), which exceeded seven (7) million subscribers in 2013. We analyze 105 (one hundred and five) questions of Physics from ENEM applied between 2009 and 2013 in an attempt to understand the scope of knowledge and the cognitive process demanded by such exam according to the Revised Bloom's Taxonomy (TBR). Among the dimensions of knowledge we highlight the Conceptual Knowledge (56\%), followed by Procedural Knowledge (31\%) and among the Cognitive Processes, what stands out is Understanding (49\%) and Application (23\%), which indicates that the exam emphasizes intermediate levels of complexity in the analyzed dimensions.

Keywords: National-Standard-High School Exam (ENEM). Revised Bloom's. Taxonomy. Physics Teaching.

\section{Vailton Afonso da Silva * Maria Inês Martins **}

\footnotetext{
* Mestre em Ensino de Ciências e Matemática. Professor de Física do Instituto Federal do Norte de Minas Gerais (IFNMG). E-mail: vailton.silva@ifnmg.edu.br

* * Doutora em Educação pela Universidade Estadual de Campinas. Professora adjunta da Pontifícia Universidade Católica de Minas Gerais. E-mail: ines@pucminas.br
} 


\section{INTRODUCุÃO}

A necessidade de se aplicar um instrumento de avaliação de aprendizagem escolar de amplitude nacional acarretou a construção de uma "matriz de referência" como referencial curricular do que será avaliado, bem como das competências e das habilidades esperadas dos alunos. A Matriz de Referência (BRASIL, 2013) consubstancia evolução importante na forma de avaliação dos estudantes e oferece orientação quanto aos conteúdos sobre os quais se espera alcançar aprendizado no Ensino Médio, pautando-se em habilidades consideradas essenciais aos estudantes que concluem esse nível de ensino.

A proposição de matrizes se tornou recorrente no Brasil desde a Lei de Diretrizes e Bases da Educação Nacional (BRASIL, 1996), a fim de induzir à reorganização curricular na Educação Básica. Exemplificam-se as matrizes, estruturadas em habilidades e competências, do Sistema Nacional de Avaliação da Educação Básica (Saeb), criado em 1997; do Exame Nacional do Ensino Médio (Enem), proposto em 1998; e do Exame Nacional para Certificação de Competências de Jovens e Adultos (Encceja), criado em 2002. Nesse contexto, o Exame Nacional do Ensino Médio (Enem) configura-se como delineador curricular e vem ganhando legitimidade no contexto escolar e na formação de professores. A evolução de inscritos no Enem é apresentada no gráfico a seguir.

Gráfico 1: Evolução de inscritos no Enem

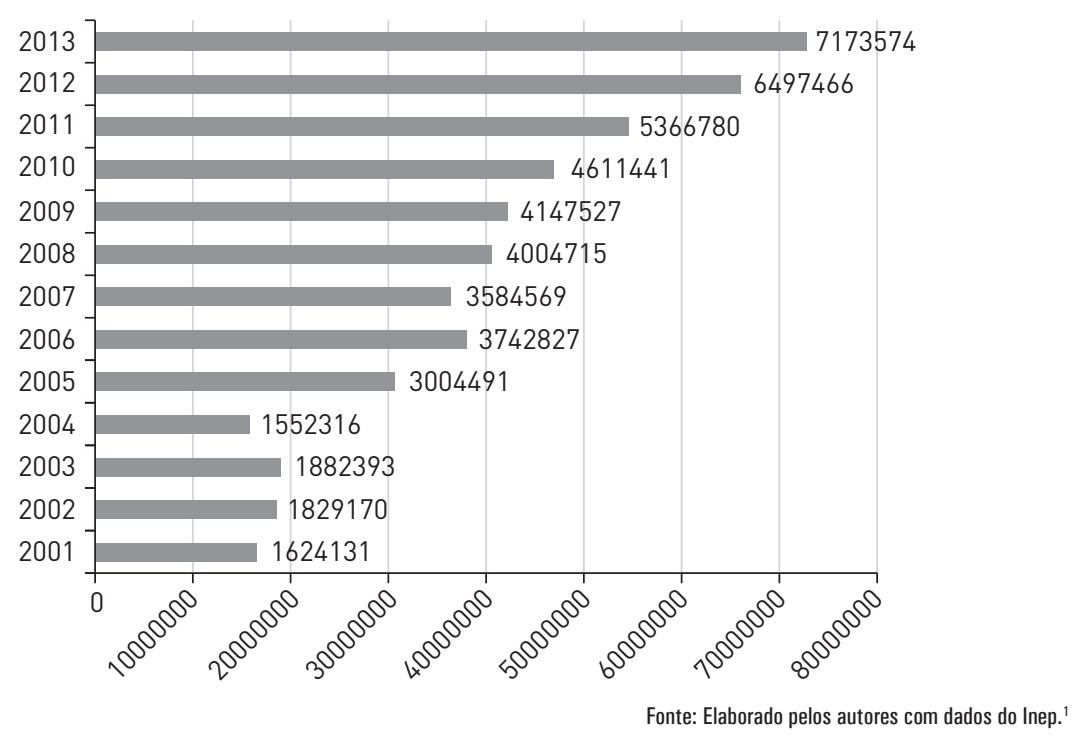

Os programas de avaliação implementados pelo governo brasileiro têm chamado a atenção para uma dimensão humana com uma longa tradição de pesquisa na psicologia: as habilidades cognitivas (ACKERMAN et al., 1999; ALMEIDA, 1988; FLANAGAN et al, 1997; STERNBERG, 1981). Nessa perspectiva, em 
1995, um grupo de especialistas encontrou-se em Syracuse, Nova York, para rever os pressupostos teóricos da Taxonomia de Bloom, uma vez que novos conceitos, tecnologias e teorias haviam sido incorporados ao campo educacional, que contava com novas publicações sobre avanços psicopedagógicos e com inúmeros trabalhos práticos. Esse grupo de especialistas (psicólogos, educadores, especialistas em currículos, testes, avaliação etc.) foi supervisionado por David Krathwohl, e, no ano 2001, o relatório dessa revisão da Taxonomia de Bloom foi publicado no livro intitulado Taxonomy for Learning, Teaching and Assessing: a revision of Bloom's taxonomy or educational objectives (ANDERSON; KRATHWOHL, 2001).

O grupo tentou buscar o equilíbrio entre a estruturação da taxonomia original e as mudanças provocadas por avanços tecnológicos e de estratégias incorporados à educação (FERRAZ; BELHOT, 2010). Os pesquisadores relacionaram os aspectos de desenvolvimento cognitivo, competência e habilidade de forma a atribuírem característica bidimensional à taxonomia original de Bloom. A partir da definição de bidimensionalidade, foram combinados o tipo de conhecimento a ser adquirido (Dimensão do conhecimento) e o processo utilizado para a aquisição desse conhecimento (Dimensão do processo cognitivo), conforme descrição sucinta apresentada nos quadros 1 e 2, a seguir:

\section{Quadro 1: Processos cognitivos na Taxonomia de Bloom Revisada (TBR)}

\section{Lembrar: Relacionado a reconhecer e reproduzir ideias e conteúdos. Reconhecer requer distinguir e selecionar uma determinada informação, e reproduzir ou recordar está mais relacionado à busca por uma informação relevante memorizada. \\ Representado pelos seguintes verbos no gerúndio: reconhecendo e reproduzindo.}

2. Entender: Relacionado a estabelecer uma conexão entre o novo e o conhecimento previamente adquirido. A informação é entendida quando o aprendiz consegue reproduzi-la com suas "próprias palavras".

Representado pelos seguintes verbos no gerúndio: interpretando, exemplificando, classificando, resumindo, inferindo, comparando e explicando.

3. Aplicar: Relacionado a executar ou usar um procedimento numa situação específica e pode também abordar a aplicação de um conhecimento numa situação nova.

Representado pelos seguintes verbos no gerúndio: executando e implementando.

4. Analisar: Relacionado a dividir a informação em partes relevantes e irrelevantes, importantes e menos importantes e entender a inter-relação existente entre as partes.

Representado pelos seguintes verbos no gerúndio: diferenciando, organizando, atribuindo e concluindo.

5. Avaliar: Relacionado a realizar julgamentos baseados em critérios e padrões qualitativos e quantitativos ou de eficiência e eficácia.

Representado pelos seguintes verbos no gerúndio: checando e criticando.

6. Criar: Significa colocar elementos junto com o objetivo de criar uma nova visão, uma nova solução, estrutura ou modelo utilizando conhecimentos e habilidades previamente adquiridos.

Envolve o desenvolvimento de ideias novas e originais, produtos e métodos por meio da percepção da interdisciplinaridade e da interdependência de conceitos.

Representado pelos seguintes verbos no gerúndio: generalizando, planejando e produzindo. 
Quadro 2: Dimensão do conhecimento na Taxonomia de Bloom Revisada (TBR)

\begin{tabular}{|c|c|c|}
\hline Categoria & Descrição & Subcategorias \\
\hline $\begin{array}{c}\text { Conhecimento } \\
\text { efetivo }\end{array}$ & $\begin{array}{l}\text { Relacionado ao conteúdo básico que } \\
\text { o discente deve dominar a fim de que } \\
\text { consiga realizar e resolver problemas } \\
\text { apoiados nesse conhecimento. Nessa } \\
\text { categoria, os fatos não precisam ser } \\
\text { entendidos ou combinados, apenas } \\
\text { reproduzidos como apresentados. }\end{array}$ & $\begin{array}{c}\text { Conhecimento da } \\
\text { terminologia; } \\
\text { conhecimento de detalhes } \\
\text { e elementos específicos. }\end{array}$ \\
\hline $\begin{array}{c}\text { Conhecimento } \\
\text { conceitual }\end{array}$ & $\begin{array}{l}\text { Relacionado à inter-relação dos } \\
\text { elementos básicos num contexto mais } \\
\text { elaborado que os discentes seriam } \\
\text { capazes de descobrir. Elementos mais } \\
\text { simples foram abordados e, agora, } \\
\text { precisam ser conectados. Esquemas, } \\
\text { estruturas e modelos foram organizados e } \\
\text { explicados. Nessa fase, não é a aplicação } \\
\text { de um modelo que é importante, mas a } \\
\text { consciência de sua existência. }\end{array}$ & $\begin{array}{c}\text { Conhecimento de } \\
\text { classificação e } \\
\text { categorização; } \\
\text { conhecimento de princípios } \\
\text { e generalizações; } \\
\text { conhecimento de teorias, } \\
\text { modelos e estruturas. }\end{array}$ \\
\hline $\begin{array}{c}\text { Conhecimento } \\
\text { procedural }\end{array}$ & $\begin{array}{l}\text { Relacionado ao conhecimento de } \\
\text { “como realizar alguma coisa” utilizando } \\
\text { métodos, critérios, algoritmos e técnicas. } \\
\text { Nesse momento, o conhecimento } \\
\text { abstrato começa a ser estimulado, mas } \\
\text { dentro de um contexto único, e não } \\
\text { interdisciplinar. }\end{array}$ & $\begin{array}{c}\text { Conhecimento de } \\
\text { conteúdos específicos, } \\
\text { habilidades e algoritmos; } \\
\text { conhecimento de técnicas } \\
\text { específicas e métodos; } \\
\text { conhecimento de critérios } \\
\text { e percepção de como } \\
\text { e quando usar um } \\
\text { procedimento específico. }\end{array}$ \\
\hline $\begin{array}{l}\text { Conhecimento } \\
\text { metacognitivo }\end{array}$ & $\begin{array}{l}\text { Relacionado ao reconhecimento da } \\
\text { cognição em geral e à consciência } \\
\text { da amplitude e da profundidade de } \\
\text { conhecimento adquirido sobre um } \\
\text { determinado conteúdo. Em contraste } \\
\text { com o conhecimento procedural, } \\
\text { esse conhecimento é relacionado à } \\
\text { interdisciplinaridade. A ideia principal } \\
\text { é utilizar conhecimentos previamente } \\
\text { assimilados (interdisciplinares) para a } \\
\text { resolução de problemas e/ou a escolha } \\
\text { do melhor método, teoria ou estrutura. }\end{array}$ & $\begin{array}{l}\text { Conhecimento estratégico; } \\
\text { conhecimento sobre } \\
\text { atividades cognitivas, } \\
\text { incluindo contextos } \\
\text { preferenciais e situações } \\
\text { de aprendizagem (estilos); } \\
\text { autoconhecimento. }\end{array}$ \\
\hline
\end{tabular}

Fonte: FERRAZ; BELHOT, 2010, p. 426.

Na nova estrutura proposta, as dimensões conhecimento e processos cognitivos foram mais claramente diferenciadas, possibilitando-se um novo modo de utilização da taxonomia, que se estrutura em um quadro bidimensional denominado Tabela Bidimensional da Taxonomia de Bloom Revisada (ANDERSON; KRATHWOH, 2001). A tabela é utilizada com o intuito de melhor definir objetivos educacionais propostos, aprimorando o planejamento e a escolha de estratégias e recursos.

A Taxonomia de Bloom Revisada (TBR) possui categorias ordenadas com certa hierarquia de complexidade e abstração, de modo que atingir uma categoria 
significa dominar as antecessoras, podendo ocorrer entrelace. Há a separação da dimensão do conhecimento (o que ensinar) da dimensão do processo cognitivo (a atividade cognitiva envolvida), possibilitando-se a criação de um esquema bidimensional. O uso da Tabela 1 possibilita verificar qual a extensão e a profundidade dos objetivos analisados e quais outros podem ser melhorados.

Tabela 1: Tabela Bidimensional da Taxonomia de Bloom Revisada

\begin{tabular}{|c|c|c|c|c|c|c|}
\hline \multirow[b]{2}{*}{ Dimensão do conhecimento } & \multicolumn{6}{|c|}{ Dimensões dos processos cognitivos } \\
\hline & $\begin{array}{l}\frac{1}{\pi} \\
\frac{0}{2} \\
\frac{E}{0} \\
\vdots\end{array}$ & 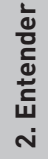 & $\begin{array}{l}\frac{1}{0} \\
\frac{0}{0} \\
\frac{0}{\alpha} \\
\text { m. }\end{array}$ & 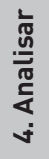 & 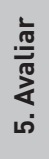 & $\begin{array}{l}\frac{1}{0} \\
\frac{1}{4} \\
0\end{array}$ \\
\hline \multicolumn{7}{|l|}{ Conhecimento efetivo / factual } \\
\hline \multicolumn{7}{|c|}{ Conhecimento conceitual / princípios } \\
\hline \multicolumn{7}{|l|}{ Conhecimento procedural } \\
\hline Conhecimento metacognitivo & & & & & & \\
\hline
\end{tabular}

Fonte: FERRAZ; BELHOT, 2010, p. 430.

Vários trabalhos analisam as questões do Enem focalizando, dentre outros aspectos, o conteúdo de física, entre os quais destacamos Hernandes e Martins (2013), que, entre os anos de 2009 e 2011, caracterizaram as questões em relação aos pressupostos dos Parâmetros Curriculares Nacionais (PCN) (BRASIL, 2000); Silva e Prestes (2009) estudaram as questões do exame relacionadas à física nas edições de 2006 a 2008; Peixoto e Linhares (2010) analisaram, na edição de 2009, o que mudou na abordagem dos conceitos de física; Silva (2013) identificou as questões de física dos anos de 2009 a 2012 por meio dos Objetos de Conhecimentos elencados no edital do Enem 2012. Por fim, o trabalho de Marcelino e Recena (2011), além de identificar as questões de química do exame referente ao ano de 2010, classificou-as na Taxonomia Bidimensional de Bloom Revisada.

O nosso trabalho, por sua vez, focaliza as questões de física do novo Enem das provas de Ciências da Natureza e suas Tecnologias ${ }^{2}$ na perspectiva da TBR, almejando classificar os níveis de abstração requeridos nessas questões e analisar as dimensões do conhecimento e do processo cognitivo da TBR privilegiadas no Enem. Entende-se que, ao serem compreendidos os níveis taxonômicos requeridos nas questões, podem-se escolher estratégias apropriadas para o ensino-aprendizagem de física no Ensino Médio. 


\section{METODOLOGIA}

Analisamos as questões dos exames de 2009 a 2013 - incluindo o Simulado do MEC/2009 e a prova anulada daquele ano - que evidenciam itens dos Objetos de Conhecimento (OC) de física, de acordo com o Edital n ${ }^{\circ} 1$, de 08 de maio de 2013 (BRASIL, 2013). Para identificação das questões de física presentes nos exames, foram utilizados os Objetos de Conhecimento elencados no referido edital e apresentados a seguir:

I. Conhecimentos básicos e fundamentais - Noções de ordem de grandeza. Notação Científica. Sistema Internacional de Unidades. Metodologia de investigação: a procura de regularidades e de sinais na interpretação física do mundo. Observações e mensurações: representação de grandezas físicas como grandezas mensuráveis. Ferramentas básicas: Gráficos e vetores. Conceituação de grandezas vetoriais e escalares. Operações básicas com vetores.

II. O movimento, o equilíbrio e a descoberta de leis físicas - Grandezas fundamentais da mecânica: tempo, espaço, velocidade e aceleração. Relação histórica entre força e movimento. Descrições do movimento e sua interpretação: quantificação do movimento e sua descrição matemática e gráfica. Casos especiais de movimentos e suas regularidades observáveis. Conceito de inércia. Noção de sistemas de referência inerciais e não inerciais. Noção dinâmica de massa e quantidade de movimento (momento linear). Força e variação da quantidade de movimento. Leis de Newton. Centro de massa e a ideia de ponto material. Conceito de forças externas e internas. Lei da conservação da quantidade de movimento (momento linear) e teorema do impulso. Momento de uma força (torque). Condições de equilíbrio estático de ponto material e de corpos rígidos. Força de atrito, força peso, força normal de contato e tração. Diagramas de forças. Identificação das forças que atuam nos movimentos circulares. Noção de força centrípeta e sua quantificação. A hidrostática: aspectos históricos e variáveis relevantes. Empuxo. Princípios de Pascal, Arquimedes e Stevin: condições de flutuação, relação entre diferença de nível e pressão hidrostática.

III. Energia, trabalho e potência - Conceituação de trabalho, energia e potência. Conceito de energia potencial e de energia cinética. Conservação de energia mecânica e dissipação de energia. Trabalho da força gravitacional e energia potencial gravitacional. Forças conservativas e dissipativas.

IV. A mecânica e o funcionamento do universo - Força peso. Aceleração gravitacional. Lei da Gravitação Universal. Leis de Kepler. Movimentos de corpos celestes. Influência na Terra: marés e variações climáticas. Concepções históricas sobre a origem do universo e sua evolução.

V. Fenômenos elétricos e magnéticos - Carga elétrica e corrente elétrica. Lei de Coulomb. Campo elétrico e potencial elétrico. Linhas de campo. Superfícies equipotenciais. Poder das pontas. Blindagem. Capacitores. Efeito Joule. Lei de Ohm. Resistência elétrica e resistividade. Relações entre grandezas elétricas: tensão, corrente, potência e energia. Circuitos elétricos simples. Correntes contínua e alternada. Medidores elétricos. Representação gráfica de circuitos. Símbolos convencionais. Potência e consumo de energia em dispositivos elétricos. Campo magnético. Imãs permanentes. Linhas de campo magnético. Campo magnético terrestre. 
VI. Oscilações, ondas, óptica e radiação - Feixes e frentes de ondas. Reflexão e refração. Óptica geométrica: lentes e espelhos. Formação de imagens. Instrumentos ópticos simples. Fenômenos ondulatórios. Pulsos e ondas. Período, frequência, ciclo. Propagação: relação entre velocidade, frequência e comprimento de onda. Ondas em diferentes meios de propagação.

VII. O calor e os fenômenos térmicos - Conceitos de calor e de temperatura. Escalas termométricas. Transferência de calor e equilíbrio térmico. Capacidade calorífica e calor específico. Condução do calor. Dilatação térmica. Mudanças de estado físico e calor latente de transformação. Comportamento de gases ideais. Máquinas térmicas. Ciclo de Carnot. Leis da termodinâmica. Aplicações e fenômenos térmicos de uso cotidiano. Compreensão de fenômenos climáticos relacionados ao ciclo da água. (BRASIL, 2013)

Depois de identificadas as questões que abordavam os Objetos de Conhecimento (OC) de física presentes nos exames, contabilizamos um total de 105 questões, que foram classificadas na Taxonomia de Bloom Revisada por meio das categorias criadas (dimensão do conhecimento versus dimensões dos processos cognitivos) numa tabela bidimensional.

\section{CLASSIFICACฺ̃̃O DAS QUESTÕES PELA TBR}

Apresentamos, a seguir, a classificação das questões de física do Enem, edições 2009 a 2013, na TBR. O número indicado corresponde à numeração do respectivo exame.

Tabela 2: Questões de física do Simulado Inep 2009 classificadas na TBR

\begin{tabular}{|c|c|c|c|c|c|c|}
\hline \multirow{2}{*}{$\begin{array}{c}\text { Dimensão do } \\
\text { conhecimento }\end{array}$} & \multicolumn{5}{|c|}{ Dimensões dos processos cognitivos } \\
\cline { 2 - 7 } & $\begin{array}{c}\text { 1. } \\
\text { Lembrar }\end{array}$ & $\begin{array}{c}2 . \\
\text { Entender }\end{array}$ & $\begin{array}{c}\text { 3. } \\
\text { Aplicar }\end{array}$ & $\begin{array}{c}\text { 4. } \\
\text { Analisar }\end{array}$ & $\begin{array}{c}\mathbf{5 .} \\
\text { Avaliar }\end{array}$ & $\begin{array}{c}6 . \\
\text { Criar }\end{array}$ \\
\hline $\begin{array}{c}\text { Conhecimento efetivo / } \\
\text { factual }\end{array}$ & & & & & & \\
\hline $\begin{array}{c}\text { Conhecimento } \\
\text { conceitual / princípios }\end{array}$ & & 6,7 & 7 & 8 & $4,6,8$ & \\
\hline $\begin{array}{c}\text { Conhecimento } \\
\text { procedural }\end{array}$ & 1 & 1 & & & \\
\hline $\begin{array}{c}\text { Conhecimento } \\
\text { metacognitivo }\end{array}$ & & & & & & \\
\hline
\end{tabular}

Fonte: Dados da pesquisa 
Tabela 3: Questões de física da prova anulada de 2009 classificadas na TBR

\begin{tabular}{|c|c|c|c|c|c|c|}
\hline \multirow{2}{*}{$\begin{array}{c}\text { Dimensão do } \\
\text { conhecimento }\end{array}$} & $\begin{array}{c}\text { 1. } \\
\text { Lembrar }\end{array}$ & $\begin{array}{c}\text { 2. } \\
\text { Entender }\end{array}$ & $\begin{array}{c}\text { 3. } \\
\text { Aplicar }\end{array}$ & $\begin{array}{c}\text { 4. } \\
\text { Analisar }\end{array}$ & $\begin{array}{c}5 . \\
\text { Avaliar }\end{array}$ & $\begin{array}{c}\text { 6. } \\
\text { Criar }\end{array}$ \\
\cline { 2 - 7 } & & 1 & & & & \\
\hline $\begin{array}{c}\text { Conhecimento } \\
\text { efetivo / factual }\end{array}$ & & $\begin{array}{c}10,13,17,19, \\
25,27,33,34, \\
35,40,42\end{array}$ & 28 & 28,40 & $\begin{array}{c}9,10,17, \\
18,19,27\end{array}$ & \\
\hline $\begin{array}{c}\text { Conhecimento } \\
\text { conceitual / } \\
\text { princípios }\end{array}$ & 23 & $\begin{array}{c}26,31,32,37, \\
44\end{array}$ & 37,44, & $23,31,32$ & & \\
\hline $\begin{array}{c}\text { Conhecimento } \\
\text { procedural }\end{array}$ & & 45 & & & & \\
\hline $\begin{array}{c}\text { Conhecimento } \\
\text { metacognitivo }\end{array}$ & & & & & & \\
\hline
\end{tabular}

Fonte: Dados da pesquisa

Tabela 4: Questões de física do Enem 2009 (prova azul) classificadas na TBR

\begin{tabular}{|c|c|c|c|c|c|c|}
\hline \multirow{2}{*}{$\begin{array}{l}\text { Dimensão do } \\
\text { conhecimento }\end{array}$} & \multicolumn{6}{|c|}{ Dimensões dos processos cognitivos } \\
\hline & $\begin{array}{c}1 . \\
\text { Lembrar }\end{array}$ & $\begin{array}{c}2 . \\
\text { Entender }\end{array}$ & $\begin{array}{c}3 . \\
\text { Aplicar }\end{array}$ & $\begin{array}{c}4 . \\
\text { Analisar }\end{array}$ & $\begin{array}{c}5 . \\
\text { Avaliar }\end{array}$ & $\begin{array}{c}6 . \\
\text { Criar }\end{array}$ \\
\hline $\begin{array}{l}\text { Conhecimento } \\
\text { efetivo / factual }\end{array}$ & & 20,24 & & 20,24 & 20 & \\
\hline $\begin{array}{c}\text { Conhecimento } \\
\text { conceitual / } \\
\text { princípios }\end{array}$ & 27 & $\begin{array}{c}5,14,18,29 \\
31,32,37 \\
39,45\end{array}$ & & 18,45 & 27,31 & \\
\hline $\begin{array}{c}\text { Conhecimento } \\
\text { procedural }\end{array}$ & & $\begin{array}{c}17,19,30 \\
35,38\end{array}$ & $\begin{array}{c}17,19,30 \\
35,38\end{array}$ & 19 & 19 & \\
\hline $\begin{array}{l}\text { Conhecimento } \\
\text { metacognitivo }\end{array}$ & & & & & & \\
\hline
\end{tabular}

Fonte: Dados da pesquisa

Tabela 5: Questões de física do Enem 2010 (prova azul) classificadas na TBR

\begin{tabular}{|c|c|c|c|c|c|c|}
\hline \multirow{2}{*}{$\begin{array}{c}\text { Dimensão do } \\
\text { conhecimento }\end{array}$} & \multicolumn{5}{|c|}{ Dimensões dos processos cognitivos } \\
\cline { 2 - 7 } & $\begin{array}{c}1 . \\
\text { Lembrar }\end{array}$ & $\begin{array}{c}2 . \\
\text { Entender }\end{array}$ & $\begin{array}{c}\text { 3. } \\
\text { Aplicar }\end{array}$ & $\begin{array}{c}\text { 4. } \\
\text { Analisar }\end{array}$ & $\begin{array}{c}5 . \\
\text { Avaliar }\end{array}$ & $\begin{array}{c}\text { 6. } \\
\text { Criar }\end{array}$ \\
\hline $\begin{array}{c}\text { Conhecimento efetivo } \\
\text { / factual }\end{array}$ & 50 & 81 & 59 & 63,89 & \\
\hline $\begin{array}{c}\text { Conhecimento } \\
\text { conceitual / princípios }\end{array}$ & $47,52,56$ & $\begin{array}{c}54,58,63,78, \\
81,84,89\end{array}$ & 70 & & & \\
\hline $\begin{array}{c}\text { Conhecimento } \\
\text { procedural }\end{array}$ & 48 & 68,70 & 70 & & & \\
\hline $\begin{array}{c}\text { Conhecimento } \\
\text { metacognitivo }\end{array}$ & & & & & & \\
\hline
\end{tabular}


Tabela 6: Questões de física do Enem 2011 (prova azul) classificadas na TBR

\begin{tabular}{|c|c|c|c|c|c|c|}
\hline \multirow{2}{*}{$\begin{array}{l}\text { Dimensão do } \\
\text { conhecimento }\end{array}$} & \multicolumn{6}{|c|}{ Dimensões dos processos cognitivos } \\
\hline & $\begin{array}{c}1 . \\
\text { Lembrar }\end{array}$ & $\begin{array}{c}2 . \\
\text { Entender }\end{array}$ & $\begin{array}{c}3 . \\
\text { Aplicar }\end{array}$ & $\begin{array}{c}4 . \\
\text { Analisar }\end{array}$ & $\begin{array}{c}5 . \\
\text { Avaliar }\end{array}$ & $\begin{array}{l}6 . \\
\text { Criar }\end{array}$ \\
\hline $\begin{array}{c}\text { Conhecimento efetivo } \\
\text { / factual }\end{array}$ & & $46,70,78,84$ & 78 & & & \\
\hline $\begin{array}{c}\text { Conhecimento } \\
\text { conceitual / princípios }\end{array}$ & & $\begin{array}{l}56,63,67 \\
74,77,86\end{array}$ & 74,77 & & & \\
\hline $\begin{array}{c}\text { Conhecimento } \\
\text { procedural }\end{array}$ & & 60,73 & 60 & & 60 & \\
\hline $\begin{array}{l}\text { Conhecimento } \\
\text { metacognitivo }\end{array}$ & & & & & & \\
\hline
\end{tabular}

Tabela 7: Questões de física do Enem 2012 (prova azul) classificadas na TBR

\begin{tabular}{|c|c|c|c|c|c|c|}
\hline \multirow{2}{*}{$\begin{array}{l}\text { Dimensão do } \\
\text { conhecimento }\end{array}$} & \multicolumn{6}{|c|}{ Dimensões dos processos cognitivos } \\
\hline & $\begin{array}{c}1 . \\
\text { Lembrar }\end{array}$ & $\begin{array}{c}2 . \\
\text { Entender }\end{array}$ & 3. Aplicar & $\begin{array}{c}4 . \\
\text { Analisar }\end{array}$ & $\begin{array}{c}5 . \\
\text { Avaliar }\end{array}$ & $\begin{array}{c}6 . \\
\text { Criar }\end{array}$ \\
\hline $\begin{array}{l}\text { Conhecimento } \\
\text { efetivo / factual }\end{array}$ & 64,67 & 67,83 & 67,83 & & & \\
\hline $\begin{array}{c}\text { Conhecimento } \\
\text { conceitual / } \\
\text { princípios }\end{array}$ & $\begin{array}{c}47,50,71 \\
84,88\end{array}$ & $\begin{array}{c}50,55,60,71 \\
73,74,78 \\
84,88\end{array}$ & $\begin{array}{l}47,55,60 \\
71,73,78\end{array}$ & 74 & & \\
\hline $\begin{array}{c}\text { Conhecimento } \\
\text { procedural }\end{array}$ & 54,72 & $54,61,72,77$ & 77 & 77 & & \\
\hline $\begin{array}{l}\text { Conhecimento } \\
\text { metacognitivo }\end{array}$ & & & & & & \\
\hline
\end{tabular}

Tabela 8: Questões de física Enem 2013 (prova branca) classificadas na TBR

\begin{tabular}{|c|c|c|c|c|c|c|}
\hline \multirow{2}{*}{$\begin{array}{l}\text { Dimensão do } \\
\text { conhecimento }\end{array}$} & \multicolumn{6}{|c|}{ Dimensões dos processos cognitivos } \\
\hline & $\begin{array}{c}1 . \\
\text { Lembrar }\end{array}$ & $\begin{array}{c}2 . \\
\text { Entender }\end{array}$ & $\begin{array}{c}3 . \\
\text { Aplicar }\end{array}$ & $\begin{array}{c}4 . \\
\text { Analisar }\end{array}$ & $\begin{array}{c}5 . \\
\text { Avaliar }\end{array}$ & $\begin{array}{c}6 . \\
\text { Criar }\end{array}$ \\
\hline $\begin{array}{l}\text { Conhecimento } \\
\text { efetivo / factual }\end{array}$ & 46,58 & 47,85 & 47,85 & & & \\
\hline $\begin{array}{c}\text { Conhecimento } \\
\text { conceitual / } \\
\text { princípios }\end{array}$ & & $\begin{array}{c}53,55,62 \\
66,68,80,88\end{array}$ & $\begin{array}{c}55,62,66 \\
68,77,80 \\
88\end{array}$ & 77 & & \\
\hline $\begin{array}{c}\text { Conhecimento } \\
\text { procedural }\end{array}$ & & $\begin{array}{l}52,72,74 \\
79,82,89\end{array}$ & $\begin{array}{l}52,72,74 \\
79,82,89\end{array}$ & 82 & & \\
\hline $\begin{array}{l}\text { Conhecimento } \\
\text { metacognitivo }\end{array}$ & & & & & & \\
\hline
\end{tabular}


A seguir, apresentamos a Tabela 9, que consolida a quantidade de questões do Enem classificadas na tabela bidimensional. Nos gráficos 2 e 3, apresentamos o consolidado quantitativo de questões, respectivamente, por dimensão do conhecimento e por níveis de cognição.

Tabela 9: Quantidade de questões de física no Enem (2009*-2013) classificadas na TBR

\begin{tabular}{|c|c|c|c|c|c|c|c|}
\hline \multirow{2}{*}{$\begin{array}{l}\text { Dimensão do } \\
\text { conhecimento }\end{array}$} & \multicolumn{7}{|c|}{ Dimensões dos processos cognitivos } \\
\hline & $\begin{array}{c}1 . \\
\text { Lembrar }\end{array}$ & $\begin{array}{c}2 . \\
\text { Entender }\end{array}$ & $\begin{array}{c}3 . \\
\text { Aplicar }\end{array}$ & $\begin{array}{c}4 . \\
\text { Analisar }\end{array}$ & $\begin{array}{c}5 . \\
\text { Avaliar }\end{array}$ & $\begin{array}{l}6 . \\
\text { Criar }\end{array}$ & TOTAL \\
\hline $\begin{array}{l}\text { Conhecimento } \\
\text { efetivo / factual }\end{array}$ & 4 & 12 & 5 & 2 & 1 & & 24 \\
\hline $\begin{array}{c}\text { Conhecimento } \\
\text { conceitual / } \\
\text { princípios }\end{array}$ & 9 & 51 & 18 & 8 & 13 & & 99 \\
\hline $\begin{array}{c}\text { Conhecimento } \\
\text { procedural }\end{array}$ & 4 & 25 & 18 & 6 & 2 & & 55 \\
\hline \multicolumn{8}{|l|}{$\begin{array}{l}\text { Conhecimento } \\
\text { metacognitivo }\end{array}$} \\
\hline TOTAL & 17 & 88 & 41 & 16 & 16 & & 178 \\
\hline
\end{tabular}

Gráfico 2: Total de questões de física por dimensão do conhecimento

Fonte: Dados da pesquisa

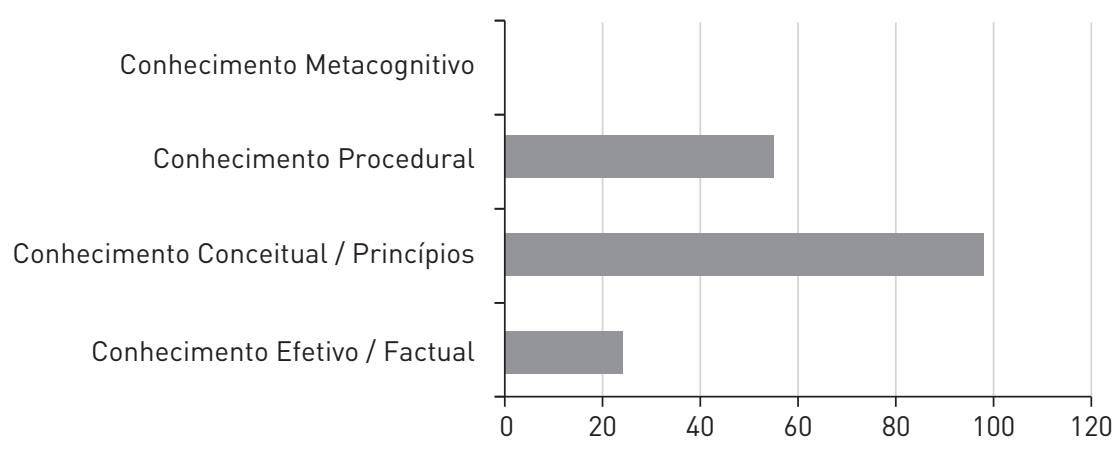


Gráfico 3: Total de questões de física por processo cognitivo Fonte: Dados da pesquisa

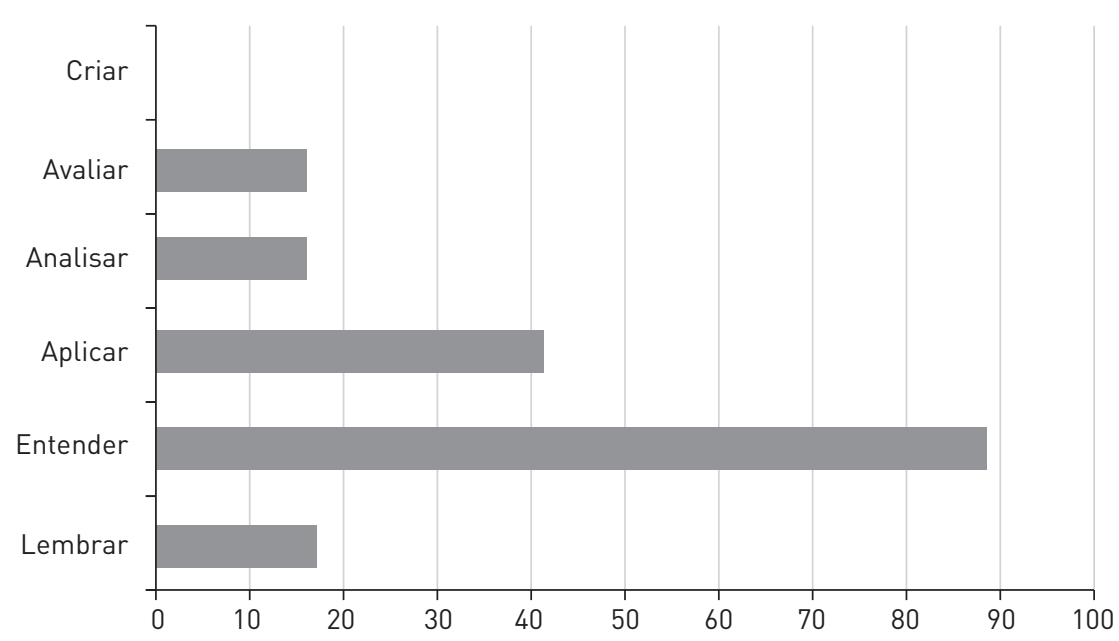

\section{ANÁLISE DOS DADOS}

Analisando as tabelas e os gráficos anteriores, tomando como referência apenas as dimensões do conhecimento, percebe-se que as questões do exame privilegiaram o conhecimento conceitual/princípios. Nessa dimensão do conhecimento, os discentes, além de dominarem os conteúdos básicos, devem fazer uma inter-relação destes conteúdos num contexto mais elaborado. Os discentes precisam conectar conhecimentos básicos, teorias, estruturas e modelos para chegar às respostas das questões que envolvem esse tipo de dimensão. A dimensão do conhecimento procedural também foi privilegiada, conforme se observa no Gráfico 2. Nessa dimensão do conhecimento, o discente deve utilizar métodos, critérios, algoritmos e técnicas sobre um determinado conteúdo específico, ou seja, deve ter habilidade e percepção de como e quando usar um procedimento específico para chegar às respostas do exame.

A dimensão do conhecimento efetivo/factual foi menos privilegiada, ou seja, o conteúdo básico que o discente deve dominar a fim de que consiga resolver as questões do exame é menos apoiado nesse conhecimento. Nessa dimensão, os conteúdos não precisam ser entendidos ou combinados, apenas reproduzidos como apresentados. Por fim, a dimensão do conhecimento metacognitivo não foi encontrada nas questões do exame. Nessa dimensão, seriam exigidos conhecimentos estratégicos, autoconhecimento e interdisciplinaridade, ou seja, um alto grau de profundidade de conhecimento e interdisciplinaridade para se resolver a questão. Acreditamos que essa dimensão do conhecimento não foi encontrada devido à limitação de tempo e de quantidade de questões abordadas no exame. 
Em relação aos processos de cognição, o nível mais privilegiado na TBR foi o entender, conforme se observa no Gráfico 3. Neste nível de cognição, os discentes devem estabelecer conexão entre o novo e o conhecimento previamente adquirido. Devem ser capazes de interpretar, classificar, inferir e comparar para poder chegar às respostas das questões do exame. O segundo nível de cognição na TBR mais exigido foi aplicar. Neste nível de cognição, é exigido dos discentes executar ou usar um procedimento numa situação nova, ou seja, utilizar o aprendizado em novas situações. Os níveis de cognição lembrar, analisar e avaliar foram igualmente cobrados; explicação sucinta destes níveis estão no Quadro 1. O nível de cognição criar não foi encontrado. Nele, o discente deve desenvolver ideias novas e originais por meio da percepção da interdisciplinaridade e da interdependência de conceitos. Em nossa pesquisa, nenhuma questão abordou esse nível de cognição, possivelmente em função da limitação do tempo e da quantidade de questões.

A análise anterior foi feita separadamente, tanto para a dimensão do conhecimento quanto para os níveis de cognição, para uma melhor interpretação do que está sendo mais exigido nas questões do exame. Como já foi dito, a TBR possui categorias ordenadas com certa hierarquia de complexidade e abstração, de modo que atingir uma categoria significa dominar as antecessoras, podendo ocorrer entrelace. Assim, numa análise geral da Tabela 7 , percebe-se que a célula com maior frequência de questões nos mostra que a ênfase nas provas do Enem são a dimensão do conhecimento conceitual e o processo cognitivo entender. Numa análise mais detalhada dessa tabela, percebemos que todas as dimensões do conhecimento, exceto o metacognitivo, perpassam os processos cognitivos de lembrar a avaliar, mas que existe um privilégio na dimensão do conhecimento conceitual em todos os processos cognitivos encontrados. É evidente o vazio na categoria de conhecimento metacognitivo em todos os níveis de cognição, assim como o vazio do nível de cognição criar em todas as dimensões do conhecimento.

A pesquisa mostra que o Enem enfatizou domínios de complexidade superiores à simples memorização, porém, sem atingir a dimensão metacognitiva, tampouco o nível de cognição criar. Tal fato reflete a Matriz de Referência do Enem, elaborada baseando-se em habilidades e competências, e não em níveis taxonômicos.

\section{CONSIDERACְÕES FINAIS}

Sugere-se a observação das competências e das habilidades presentes na Matriz de Referência do Novo Enem procurando-se, a partir delas, construir um planejamento com base na Taxonomia Revisada de Bloom, o que facilita a definição clara de objetivos de aprendizagem, alinhando-os às atividades de avaliação. Salienta-se que, no planejamento, não há problema algum na ordem dos objetivos inseridos na dimensão dos processos cognitivos, entretanto, a ordem na dimensão 
de conhecimentos deve ser respeitada de forma hierárquica e almejando um maior controle do processo de aprendizagem. Por fim, sugere-se a inexistência de colunas em branco.

Existem modelos de planejamento com base na Taxonomia Bidimensional de Bloom que podem servir de exemplo. Ferraz e Belhot (2010) sugerem um modelo que pode ser seguido pelos professores. Os autores destacam que planejar uma disciplina ou um curso não é tarefa fácil, sobretudo para profissionais sem preparo didático nem pedagógico, realidade que muitos docentes enfrentam com regularidade.

A não realização de um planejamento pedagógico adequado, que delimite o conteúdo, com escolhas e estratégias educacionais eficazes, pode redundar em alto grau de evasão ou mesmo em frustração docente diante de discentes que não atingem o nível de desenvolvimento (cognitivo, de competência e de habilidade) desejado. Tais resultados podem ser fruto da falta de comprometimento efetivo dos discentes, mas também podem responder às dificuldades enfrentadas na realização das tarefas propostas por falta de compreensão adequada do objetivo pretendido, da importância do conteúdo abordado e das técnicas instrucionais utilizadas, além da sua adequação aos critérios de avaliação e de recuperação do aprendizado.

Todo desenvolvimento cognitivo deve seguir uma estrutura hierárquica, para que, no momento oportuno, os discentes sejam capazes de aplicar e transferir, de forma multidisciplinar, um conhecimento adquirido. Nessa perspectiva, o planejamento é essencial e precisa ser estruturado de forma coerente, tanto com base em objetivos bem-definidos (gerais e específicos), na delimitação dos conteúdos, na escolha das estratégias e dos instrumentos de avaliação quanto para "medir" o que foi aprendido e direcionar, de forma corretiva e formativa, o processo educacional.

A utilização de instrumentos que facilitem esse processo de planejamento é fundamental, e, nesse contexto, a Taxonomia de Bloom Revisada tem colaborado significativamente, pois é um instrumento de classificação de objetivos de aprendizagem de forma hierárquica (do mais simples para o mais complexo) que pode ser utilizado para estruturar, organizar e planejar disciplinas, cursos ou módulos instrucionais.

\section{NOTAS}

\footnotetext{
${ }^{1}$ Fonte: www.inep.gov.br

${ }^{2}$ Foram analisadas as edições do novo Enem de 2009 a 2013, além do simulado divulgado pelo Inep e da prova anulada de 2009.
} 


\title{
REFERÊNCIAS
}

ACKERMAN, P.L. et al. Learning and individual differences. Washington, DC: American Psychological Association, 1999.

ALMEIDA, L. S. Teorias da Inteligência. Porto: Edições Jornal de Psicologia, 1988.

ANDERSON, L. W.; KRATHWOHL, K. R. A. Taxonomy for Learning, Teaching and Assessing: a revision of Bloom's taxonomy or educational objectives. New York: Longman, 2001.

BRASIL. Lei no 9.394, de 20 de dezembro de 1996. Diretrizes e Bases da Educação Nacional. Diário Oficial da União. Brasília, 23/12/1996.

- Ministério da Educação. Instituto Nacional de Estudos e Pesquisas Educacionais Anísio Teixeira. Edital $n^{0}$ 01, de 08 de maio de 2013. Exame Nacional do Ensino Médio - ENEM 2013. Brasilia: MEC/Inep, 2013.

. Ministério da Educação, Secretaria de Educação Média e Tecnológica. Parâmetros Curriculares Nacionais para o Ensino Médio. PCNEM. Brasília: MEC/Semtec, 2000.

FERRAZ, A. P. C. M.; BELHOT, R. V. Taxonomia de Bloom: revisão teórica e apresentação das adequações do instrumento para definição de objetivos instrucionais. Gestão \& Produção. [online]. v.17, n.2, p. 421-431, 2010. Disponível em: <http://dx.doi.org/10.1590/S0104-530X2010000200015>. Acesso em: 04 out. 2013.

FLANAGAN, D. P. et al. Contemporary intellectual assessment. theories, tests, and issues. New York: The Guilford Press, 1997.

HERNANDES, J. S.; MARTINS, M. I. Categorização de questões de Física do Novo Enem. Caderno Brasileiro de Ensino de Física, Florianópolis, v. 30, n. 1, p. 58-83, 2013.

MARCELINO, L. V.; RECENA, M. C. P. Análise de questões do novo Enem segundo a Taxonomia Revisada de Bloom. In: REUNIÃO ANUAL DA SOCIEDADE BRASILEIRA DE QUÍMICA, 34., 2011. Florianópolis. [Resumos]. Florianópolis: SBQ, 2012. Disponível em: <www.sbq.org.br/ 34ra/34RASBQ.pdf>. Acesso em: 22 nov. 2012.

PEIXOTO, K. C. Q.; LINHARES, M. P. Novo Enem: o que mudou? Uma investigação dos conceitos de Física abordados no Exame. In: ENCONTRO DE PESQUISA E ENSINO DE FÍSICA, 12. 2010, Águas de Lindoia. Anais... São Paulo: SBF, 2010.

SILVA, A. M. M.; PRESTES, R. F. Conhecimentos de Física nas questões do Enem. In: SEMINÁRIO NACIONAL DE ENSINO DE FÍSICA, 18., 2009, Vitória. Anais... São Paulo: SBF, 2009.

SILVA, V. A. Questões do Enem nos livros didáticos de Física recomendados pelo PNLD 2012. 2013. $161 \mathrm{f}$. Dissertação (Mestrado em Ensino de Ciências e Matemática) - Pontifícia Universidade Católica de Minas Gerais, Belo Horizonte.

STERNBERG, R.J. The evolution of theories of intelligence. Intelligence, v.5, p. 209-230, 1981.

\section{Informação}

A pesquisa retratada no artigo foi desenvolvida em conjunto pelos dois autores.

Data Recebimento: 07/05/2014

Data Aprovação: 11/11/2014

Data Versão Final: 09/12/2014

\author{
Contato: \\ Vailton Afonso da Silva \\ Instituto Federal de Educação Ciência e Tecnologia do Norte de Minas Gerais. \\ Fazenda Varginha Km 02 Rod.Salinas/Taiobeiras \\ Centro - Salinas, MG - Brasil \\ CEP: 39560000. Email: vailton.silva@ifnmg.edu.br
}

